\section{Deubiquitylating Enzymes}

\author{
Xin $\mathrm{Li}^{1}$ and Q. Ping Dou ${ }^{2}$ \\ ${ }^{1}$ School of Food and Biological Engineering, \\ Guangdong Polytechnic of Science and Trade, \\ Guangzhou, China \\ ${ }^{2}$ Barbara Ann Karmanos Cancer Institute, \\ Departments of Oncology, Pharmacology and \\ Pathology, School of Medicine, Wayne State \\ University, Detroit, MI, USA
}

\section{Synonyms}

Deubiquitinases

\section{Definition}

Deubiquitylating enzymes (DUBs) are the peptidases that can remove ubiquitin from substrate proteins, edit ubiquitin chains, or cleave ubiquitin precursors. Human genome encodes about 100 DUBs that can be classified into six families based on similar sequence and conserved domains. These DUB families include ubiquitin-specific proteases (USPs), ubiquitin carboxyl-terminal hydrolases (UCHs), Machado-Josephin domain-containing proteases (MJDs), ovarian tumor proteases (OTUs), motif interacting with ubiquitin-containing novel DUB family (MINDYs), and JAB1/MPN/MOV34 metalloenzyme family (JAMMs). The first five DUB families are cysteine peptidases with the catalytic triad of cysteine, histidine, and asparagine/asparagine residue, whereas JAMMs are zinc metallopeptidases. DUBs exhibit strong substrate selectivity in targeting individual proteins and structural motifs. This specificity is imparted via the key domain of DUBs, i.e., a zinc finger ubiquitin-specific protease domain (ZnF-UBP domain) (Dou and Zonder 2014; Farshi et al. 2015; Harrigan et al. 2018).

\section{Basic Characteristics}

\section{Characteristics of Ubiquitylation}

Ubiquitylation exhibits vital enzymatic reactions for post-modification and turnover of substrate proteins in cellular processes. Ubiquitin, a 76-residue polypeptide, is conjugated to different residues of a variety of target proteins. In humans, approximately 600 ubiquitin E3 ligases have been identified to ensure the specificity of substrate selection. Linear ubiquitin chains exist in several formats depending on the linkage of the internal ubiquitin lysine residue (Lys6, Lys11, Lys27, Lys29, Lys33, Lys48, or Lys63). Similarly, polyubiquitin chains have different linkages that are involved in linkages between ubiquitin and ubiquitin-like proteins (UBLs) such as small ubiquitin-like modifier (SUMO) and neuronal precursor cell-expressed developmentally downregulated protein 8 (NEDD8). Based on different types of ubiquitin and UBL modifications, the target proteins exhibit specific and diverse effects on cellular physiology. Such modifications of 
target protein are found in the ubiquitin proteasome system (UPS). Mostly, proteins polyubiquitylated via E3 ligases are recognized and then degraded by multimeric $26 \mathrm{~S}$ proteasome complex; thus the ubiquitin moieties are recycled by the UPS. Currently, protein ubiquitylation process commonly exists in eukaryotic cells and is well investigated. Ubiquitylation plays a central role in regulating protein interactions, localization, and enzymatic activities and is involved in regulating DNA transcription, DNA damage and repair, cell cycle progression, endocytosis, apoptosis, and other cellular processes (Farshi et al. 2015).

\section{Characteristics of Deubiquitylating Enzymes}

Protein ubiquitylation is a reversible process conducted by DUBs. Just like phosphatases reversing protein phosphorylation, DUBs trim or remove ubiquitin chains linked to the substrate protein, act as vital regulatory components for posttranslational modifications, and add to the complexity and sensitivity of the UPS. The complex nature of UPS modulation helps the cell to accurately respond to various physiological states and stimuli through modulating the stability of key molecules in the cells.

DUBs selectively cleave ubiquitin chains to release ubiquitin. Through this critical process, DUBs modulate the degradation or activation of target proteins. Thus, diverse processing or removal of polyubiquitin moieties or chains on proteins regulated by DUBs determines the fate of targeted proteins into degradation by proteasome or another function (such as traveling to a differential subcellular localization). Importantly, DUB-mediated cleavage of polyubiquitylated chains works in a similar manner as a protein phosphatase to release phosphorylated groups in the kinase-phosphatase network. Thus, DUBs and E3 ligases determine protein fates in a similar way that kinases and phosphatases regulate protein activities. Specific cleavage along the ubiquitin chains in different locations can have differential effects on the fates of ubiquitylated proteins, which provides the cells with a complex and dynamic system to modulate protein turnover, activity, expression, and localization. In addition, DUBs regulate gene expression, apoptosis, cell cycle, DNA repair, and signaling (Harrigan et al. 2018).

\section{DUBs in Cellular Signaling}

\section{Regulation of p53 Signaling}

Deubiquitylation has emerged as a crucial process of p53 stability regulation. Several DUBs are involved in modulating the E3 ligase MDM2 (Murine double minute 2) that is a negative regulator of p53 stability. For example, OTUB1 can abrogate p53 ubiquitylation and increase p53 stability and activity via inhibiting MDM 2 cognate ubiquitin-conjugating enzyme (E2) UbcH5. Through binding to E2 enzyme Ubc13, OTUB1 also modulates the K63-ubiquitylated chromatin induced by DNA damage. By cleaving ubiquitin chains on MDM2, USP7 can inhibit proteasomal degradation of MDM2 protein, leading to accumulation of MDM2 and suppression of p53 through increased ubiquitylation and degradation of p53. So, USP7 inhibition should trigger MDM2 degradation, p53 stabilization, and ultimately activation of apoptotic pathways in tumor cells. USP42 is another DUB important for p53 stability. p53 can be directly deubiquitylated by USP42 that reverses its ubiquitylation by MDM2. USP42 forms a direct complex with p53 and controls its activation in response to cellular stress; as a result it regulates p53-dependent transcription and cell cycle arrest. In addition, other DUBs such as USP2 and USP15 have been characterized to bind with $\mathrm{p} 53$ and deubiquitylate MDM2, thereby impeding expression of p53 (Harrigan et al. 2018).

\section{Regulation of NF- $\kappa B$ Signaling}

Ubiquitylation of key signaling molecules by E3 ubiquitin ligases has emerged as an important regulatory mechanism for nuclear factor-kappa $\mathrm{B}(\mathrm{NF}-\kappa \mathrm{B})$ signaling. DUBs counteract E3 ligases and therefore play a crucial role in the downregulation of NF- $\mathrm{KB}$ signaling and homeostasis. An important molecule A20, which was first identified as a zinc finger protein, can negatively regulate NF- $\kappa \mathrm{B}$ signaling pathway by modifying 
receptor-interacting protein (RIP) with K63-ubiquitin chain and targeting RIP for degradation. Further investigating the mechanisms of NF- $\kappa \mathrm{B}$ downregulation by specific DUBs such as A20 and CYLD (cylindromatosis) may provide therapeutic opportunities for the treatment of chronic inflammatory diseases and cancer (Harhaj and Dixit 2011).

\section{Regulation of TGF- $\beta$ Pathway}

Transforming growth factor- $\beta$ (TGF- $\beta$ ) pathway has a tumor suppressor role in normal and premalignant cells but promotes oncogenesis in advanced cancer cells. Several DUBs like USP4, USP11, USP15, USP18, USP9X, A20, AMSH, CYLD, and $\mathrm{UCH} 37$ can regulate TGF- $\beta$ signaling pathway. UCH37 interacts with SMAD7 (mothers against decapentaplegic homolog 7) and deubiquitylates T $\beta R-I$ (transmembrane serine/ threonine kinase receptors type I) and therefore stabilizes the type I receptor and acts as the counterpart of SMAD ubiquitin regulatory factors (SMURFs) in regulating expression of T $\beta R$-I. $\mathrm{UCH} 37$ also increases early signaling and plays a role in TGF- $\beta$-induced migration. USP 15 binds to the SMAD7-SMURF2 complex and deubiquitylates and stabilizes the type I TGF- $\beta$ receptor, leading to enhanced TGF- $\beta$ signaling. USP15 in glioblastoma has been identified to stabilize T $\beta$ R-I and activate oncogenesis through the activation of TGF- $\beta$ signaling. In addition, USP15 regulates TGF- $\beta$ signaling by deubiquitylating mono-ubiquitylated receptor-regulated SMADs (R-SMADs) as well. CYLD acts as an inhibitor against JNK (c-Jun amino-terminal kinase) and NF-kB signaling and regulates TGF- $\beta$ signaling in $\mathrm{T}$ cells. CYLD preferentially hydrolyzes or cleaves K63-linked ubiquitin chains and regulates TGF- $\beta$ signaling. CYLD inhibits the TGF- $\beta$-induced development of regulatory $\mathrm{T}$ cells through deubiquitinating SMAD7 that inhibits the activation of transforming growth factor receptor- $\beta$-activated kinase 1 (TAK1) and $\mathrm{p} 38$. AMSH (associated molecule with the $\mathrm{SH} 3$ domain of STAM) and AMSH-LP (AMSH-like protein) bind to inhibitory SMADs (I-SMADs) and inhibit their function to enhance TGF- $\beta$ and bone morphogenetic protein (BMP) signaling.
Mono-ubiquitylation of SMAD4 at K519 blocks binding of SMAD4 to phospho-SMAD2; FAM/USP9 $x$ reverts this negative modification and re-empowers SMAD4 function. These findings provide a novel molecular target for the design of inhibitors with therapeutic potential in various diseases (Iyengar 2017).

\section{Cytokine-Inducible Deubiquitinating Enzymes}

Currently, the well-characterized cytokine-inducible DUBs in murine include DUB-1, DUB-1A, DUB2, DUB-2A, and DUB-3, which exert regulatory functions in cell proliferation and apoptosis in lymphocytes. For example, IL-3, IL-5, and GM-CSF stimulate DUB-1 expression, thereby arresting growth in the $\mathrm{G}_{1}$ phase of the cell cycle. Essentially, the inhibitory effect of DUB-1 is specific to hematopoietic cells. Like DUB-1, DUB-2 is also stimulated by IL-2 and expressed in T lymphocytes that exhibited constitutive activation of the IL-2 signaling pathway. Interestingly, expression of DUB-2 in $\mathrm{Ba} / \mathrm{F} 3$ cells can enhance the survival of these cells through strongly inhibiting apoptosis induced by cytokine withdrawal. In addition, DUB-3 induced by IL-4 and IL- 6 can regulate cell growth and survival and, when constitutively expressed, results in growth suppression and apoptosis. Since cytokineinducible DUBs play the vital role in various immune-related cells, it might be interesting to investigate cytokine-inducible human DUB homologues and define cytokine signal transduction and molecular mechanisms through the discovery of DUB inhibitors (Lim et al. 2013).

\section{DUBs as Clinical Markers of Cancers}

Several mutated DUBs serve as oncogenes (e.g., USP6 and USP28) or tumor suppressors (e.g., CYLD and A20). Moreover, activities and expression levels of DUBs frequently alter in a variety of malignant tumors. These findings exhibit the clinical relevance of DUBs in cancer development and offer valuable markers for predicting responses of cancer patients to chemotherapeutic drugs.

Somatic mutations in USP6 and USP28 have been detected in various human malignancies. For 
example, the overexpression of USP6 (Tre-2) is triggered by chromosomal rearrangements in the osteoblast cadherin 11 gene (CDH11) promoter region, which turns USP6 into an oncogene associated with neoplastic aneurismal bone cysts. Mutated USP28 and CYLD are widely found in cases of lobular breast cancer and familiar cylindromatosis, respectively. In several lymphoma subtypes, the chromosomal deletions and inactivating mutations of A20 have been detected as well.

A variety of DUBs are linked to cancer through altered expression patterns. For instance, the DUB dysregulation of human cancers can be screened through in situ hybridization on tissue microarrays. In non-small cell lung carcinomas, the significantly elevated expression levels of JOSD1, CSN5, UCHL1, and USP9X are observed, whereas USP10, USP11, USP22, USP48, and CSN5 are upregulated in malignant melanoma. USP10, USP11, and USP22 levels are significantly higher in metastatic melanoma compared with benign nevi and primitive tumors. Therefore, these DUBs might offer useful indicators for diagnostic/prognostic evaluation or as new therapeutic targets. Similarly, USP22 is overexpressed in several cancers including colorectal carcinomas, liver and breast cancer, as well as oral squamous carcinoma and esophageal squamous cell carcinoma; therefore this DUB serves as a new molecular marker to predict the prognosis of human metastatic cancers. Interestingly, USP2 expression is downregulated in breast carcinomas. Conversely, USP2 is overexpressed in ovarian and prostate carcinomas and is associated with lesions of poor prognosis. Moreover, overexpression of USP2 protects prostate cancer cells from apoptosis and confers them resistance to chemotherapeutic agents through decreasing p53 stability, suggesting that USP2 has dual properties (protumor or antitumor) exhibited in a tissuespecific manner. USP8 levels are upregulated in cervical squamous cell carcinoma (CSCC) tissue samples compared to healthy tissues. Importantly, elevated levels of USP8 promote cell proliferation, migration, and invasion of CSCC cell lines. Thus, high expression of USP8 correlates with tumor stage and might be used as an independent prognostic marker for CSCC. USP7 overexpression in prostate cancer is also connected with tumor aggressiveness. USP4 is overexpressed in several types of human cancer, but downregulated in small-cell lung cancer cell lines. Moreover, elevated expression levels of USP17 have been identified in primary lung, colon, esophagus, and cervix tumor biopsies. Furthermore, USP15 is downregulated in paclitaxelresistant ovarian cancer, and reduced expression of CYLD is found in melanoma and other malignant tumors. USP28 can stabilize the levels of Myc through an interaction with FBW7 $\alpha$ (F-box and WD40 domain-containing protein $7 \alpha$ ) and is highly expressed in colon and breast carcinoma. In addition, USP14 expression is high in different cancers including ovarian and colorectal cancer. The upregulated USP14 expression levels in colorectal cancer are associated with the pathologic stages as well as liver and lymph node metastases. CXCR4 (C-X-C motif chemokine receptor 4) degradation and chemotaxis are controlled due to its deubiquitylation by USP14. In gastric cancer, USP14 also serves as a novel prognostic indicator to encourage cisplatin resistance via Akt/ERK (serine-threonine kinase/extracellular signal-regulated kinase) signaling pathways (Fraile et al. 2012; Harrigan et al. 2018).

In addition, DUBs serve as the key regulatory components in various tumor metastatic events. These events in the metastatic progression include epithelial mesenchymal transition (EMT), the extracellular matrix (ECM) degradation, cell fate regulation, as well as resistance of apoptosis in the circulation. Currently, several well-characterized DUBs such as USP4, UCHL1, UCHL3, USP22, and USP47 are involved in modulating EMT process. USP6, USP22, USP2, and USP42 participate in matrix metalloproteinase (MMP) regulation as well. USP21, USP13, USP1, PSMD14, USP7, USP15, and USP21 are important regulatory DUB members found in cell fate determinants. Furthermore, DUBs like USP9X and ataxin-3 exhibit regulatory activities in cell apoptosis process (detailed review in citation (He et al. 2017)). 


\section{DUBs in Immunity and Inflammation}

Mutations in specific DUBs are associated with chronic inflammation, autoimmunity, infectious disease, cancer, and neurodegeneration. Several DUBs such as A20, CYLD, and OTULIN are well characterized as vital regulators of inflammatory signaling and cell death (Harrigan et al. 2018).

A20 exhibits negative feedback of NF- $\kappa$ B activation in response to various proinflammatory stimuli including TNF, IL-1, LPS (lipopolysaccharide), T-cell and B-cell receptor antigens (e.g., pathogens), and nucleotide-binding oligomerization domain-containing protein 2 (NOD2) ligands. A20 specifically removes K63-linked polyubiquitylation chains from multiple $N F-\kappa B$ signaling factors including $N F-\kappa B$ essential modulator (NEMO), receptor-interacting serine/threonine protein kinase 1 (RIPK1), and tumor necrosis factor (TNF) receptor-associated factor 6 (TRAF6), thereby restricting the Toll-like receptor (TLR) signaling and maintaining immune homeostasis. In addition, A20 binds to polyubiquitin chains via its seventh zinc finger motif $(\mathrm{ZnF} 7)$ and then is recruited to NEMO. This A20-NEMO interaction impairs the I $\mathrm{B}$ kinase (IKK) phosphorylation by its upstream kinase TAK1, thereby reducing NF- $\kappa \mathrm{B}$ activation. Furthermore, A20 exerts crucial roles in controlling inflammatory responses and might be an important determinant for multiple autoimmune diseases. In macrophages, A20 regulates IL-1 $\beta$ / IL-18 release by controlling NLRP3 (nucleotidebinding domain, leucine-rich-containing family, pyrin domain-containing-3) inflammasome activity and CXCL9/CXCL10 (C-X-C motif chemokine 9/10) production through STAT1 (signal transducer and activator of transcription 1) signaling. In essential, the autoinflammatory arthritis phenotype is restricted by these pathways. In dendritic cells, A20 regulates the expression of co-stimulatory molecules (e.g., CD80/CD60), proinflammatory cytokines (e.g., IL-6), and antiapoptotic proteins, thereby exhibiting in vivo T-cell and B-cell homeostasis. A20 regulates necroptosis and autophagy in $\mathrm{CD}^{+} \mathrm{T}$ cells, as well as necroptosis, IL-2, and IFN $\gamma$ release in
$\mathrm{CD}^{+} \mathrm{T}$ cells. Furthermore, A20 perturbs regulatory T-cell development. A20 in B cells governs co-stimulatory molecule expression, IL-6 production, and Bcl-x survival protein expression, thereby blocking autoreactive B-cell generation leading to an autoimmune phenotype such as systemic lupus erythematosus.

CYLD is a negative regulator of RIG1 (retinoic acid-inducible gene-1-like receptor 1)-mediated antiviral response. CYLD can bind to RIG1 and thereby inhibit ubiquitylation and signaling functions of RIG1. CYLD also inhibits the ubiquitylation of TBK1 (TANK-binding kinase 1) and $\mathrm{IKK} \varepsilon$, which are implicated in the negative regulation of IFN responses. In dendritic cells, CYLD deficiency triggers constitutive activation of TBK1 and IKKE. Furthermore, CYLDdeficient cells and mice are more sensitive to viral infection due to downregulated interferon (IFN) receptor signaling and antiviral gene expression stimulated by IFN $\beta$. Interestingly, CYLD-deficient mice exhibit disorder of thymocyte development and activation of T cells associated with bowel inflammation and autoimmune responses. In essential, CYLD interacted with lymphocyte-specific protein tyrosine kinase (LCK). The active LCK is recruited to its substrate, zeta-chain-associated protein kinase 70 (ZAP70), and then activates downstream T-cell receptor (TCR) signaling events. Also, CYLD exerts key regulatory effects on B-cell function. Lack of CYLD expression can induce constitutive activation of the canonical NF-kB signaling cascade in B cells. Furthermore, CYLDdeficient B cells exhibit defects in B-cell maturation and homeostasis. These defects are characterized by hyperproduction of marginal zone B cells and expansion of B cells in peripheral lymphoid organs. Similarly, abnormalities of B-cell function have been observed in CYLD ${ }^{\text {ex7/8 }}$ knock-in mice with a shorter isoform of CYLD (sCYLD).

Modification of proteins with M1-linked polyubiquitin chains exerts important roles in regulating immune and inflammatory signaling cascades. M1-linked polyubiquitin chains can form from the linear ubiquitin chain assembly complex (LUBAC). This assembly complex consists of heme-oxidized IRP2 ubiquitin ligase 1 (HOIL1, 
also known as RBCK1), HOIL1-interacting protein (HOIP, also known as RNF31), and SHANKassociated RH domain-interacting protein (SHARPIN). Importantly, LUBAC can be recruited to a wide range of immune receptors and ubiquitylate target proteins, especially RIPK1, RIPK2, MYD88, interleukin-1 receptorassociated kinases (IRAKs), and NEMO. Furthermore, deficiency of M1-linked ubiquitin chains perturbs immune signaling in LUBAC component-deficient mice. To date, OTULIN has been identified to specifically cleave M1 linkages. Knockdown of OTULIN results in M1-ubiquitinated protein accumulation. Conversely, overexpression of OTULIN attenuates TNF-induced NF- $\kappa \mathrm{B}$ activation and proinflammatory signaling. Furthermore, phosphorylation-dependent regulation of interaction between OTULIN and HOIP governs the functions of OTULIN and offers a subtle control for M1-linked polyubiquitin signaling. The roles of A20, CYLD, and OTLIN in NF- $\kappa$ B signaling and cell death are so similar, but the nonredundant functions of these DUBs are determined by the cellular context. The cross talk between these DUBs needs to be further investigated.

In addition, USP4 can stabilize the nuclear receptor retinoid-related orphan receptor $\gamma \mathrm{t}$ (ROR $\gamma \mathrm{t}$ ) in TH17-activated T cells and offer a potential therapeutic target for rheumatoid arthritis. In dendritic cells, TRABID is required for TLR-mediated expression of the inflammatory cytokines IL-12 and IL-23. USP18 regulates the TAK1-TAB interaction, which is required for TH17 cell differentiation and the autoimmune response. The DUB cellular zinc finger anti-NF$\kappa \mathrm{B}$ protein (CEZANNE, also known as OTUD7B) is participant in T-cell receptor signaling. It binds to ZAP70, thereby perturbing the interaction of ZAP70 with negative regulatory phosphatases. USP10 can deubiquitylate and stabilize T-bet, leading to enhanced secretion of IFN $\gamma$. Moreover, elevated expression of USP10 is found in peripheral blood mononuclear cells from patients with asthma compared with healthy donors. Thus, the expression of DUBs in various immune cells will be a new area for further investigation.

\section{DUBs in Infectious Diseases}

DUBs can function as potential therapeutic targets for various infectious diseases including viral infections, bacterial infections, and parasitic infections (Harrigan et al. 2018). For example, both severe acute respiratory syndrome coronavirus (SARS-CoV) and Middle East respiratory syndrome coronavirus (MERS-CoV) are highly pathogenic human coronaviruses, which contain papain-like cysteine proteases termed SARS-CoV PLpro and MERS-CoV PLpro, respectively. These proteases have structural similarity to the USP family of DUBs based on their X-ray structures. SARS-CoV papain-like proteases (PLPs) negatively regulate antiviral defenses by disrupting the STING (stimulator of interferon genes)-mediated IFN induction. Bacterial effectors often target kinase cascades involved in inflammation or the eukaryotic Ub system to promote bacterial pathogenicity. DUBs in Shigella (ShiCE) and Rickettsia (RickCE) showed strong preference for the K63-linked ubiquitin chains. Therefore, bacterial DUBs are potential therapeutic targets. The deubiquitylating enzyme PfUCH54 identified from Plasmodium falciparum has deubiquitylating activity and can remove adducts of the UBL NEDD8. Similarly, PfUCHL3 identified from Toxoplasma gondii has the dual specificity of the enzyme that is orthologous to mammalian UCHL3 and found to be required for parasite survival. Taken together, it will be interesting to further investigate DUB inhibitors with anti-infective activities.

\section{Drugs}

The development of selective small molecule inhibitors that targeted DUBs might be encouraging for anticancer therapeutics. Bortezomib is the first $20 \mathrm{~S}$ proteasome inhibitor (PI) approved by the FDA in 2003 for the treatment of multiple myeloma (MM) and mantle cell lymphoma. However, the extended treatments of bortezomib result in several adverse effects due to its nonselectivity. Researchers have been looking for strategies to improve PI-based therapies. 
A variety of DUBs such as USP4 (UNP), USP6 (Tre-2), USP8 (UBPY), USP14, USP28, and UCHL5 (UCH37) have been found to function as crucial modulators in cancer development and progression. Moreover, the crystal structures of USP and UCH subfamilies (e.g., USP2, USP7, USP8, USP14, and USP21) have been well characterized, thereby attracting molecular recognition profiling investigations of these DUBs in their active states. These types of investigations have helped the rationale to design and develop DUB inhibitors. Thus, targeting specific upstream components of the UPS such as DUBs and E3s will be able to improve the current therapeutic treatments for cancer and other diseases (Dou and Zonder 2014; Farshi et al. 2015; Fraile et al. 2012; Harrigan et al. 2018; Komander et al. 2009; Ndubaku and Tsui 2014).

A small molecule PR-619 with moderate inhibitory activity is selective for DUBs over other cysteine proteases. HBX 41,108 originally identified as a USP7 inhibitor is a nonselective DUB inhibitor. HBX 41,108 can stabilize p53 in HEK293 cells and lead to caspase-3 and poly (ADP-ribose) polymerase (PARP) cleavage in both $\mathrm{p} 53 \mathrm{p} / \mathrm{p}$ and $\mathrm{p} 53 / \mathrm{HCT}-116$ cells. But HBX 41,108 can also inhibit USP5, USP8, UCH-L3, and caspase- 3 with a potency $(70-200 \mathrm{nmol} / \mathrm{L})$ greater than its activity against USP7 $(530 \mathrm{nmol} /$ $\mathrm{L})$. Considering the key regulatory roles played by USP7 in the cell physiological processes, there is growing interest in investigating USP7-specific inhibitors. The novel small molecule USP7specific inhibitor P5091 identified by Progenra can stabilize p53, can impede cancer cell proliferation, and is also an effective antitumor agent in various tumor models. For example, P5091 can trigger cell apoptosis in multiple myeloma cells resistant to conventional and bortezomib therapies. Interestingly, P5091 is well-tolerated in animals, inhibits tumor growth, prolongs survival, and enhances synergistic anti-multiple myeloma activity in combination with other chemotherapeutic agents such as lenalidomide, histone deacetylase (HDAC) inhibitor, or dexamethasone. Hit-to-lead optimization identified additional analogs of P5091 (e.g., aqueous soluble derivative P045204) that increases the steady-state levels of p53 and its transcriptional target p21 in a timedependent manner in HCT-116 cells.

b-AP15 (or VLX1500) is characterized as a member of a unique class of proteasome inhibitors. It has potent inhibitory effects on the $19 \mathrm{~S}$ regulatory particle-associated UCHL5 and USP14. Importantly, b-AP15 triggers apoptosis in tumor cells regardless of their p53 or BCL2 accumulation and potently blocks tumor progression and dissemination in multiple solid tumor mouse models and an in vivo AML model.

The molecules like pimozide, GW7647, and ML323 are potent inhibitors of the USP1-UAF1 complex. Pimozide or GW7647 can noncompetitively bind with the USP1-UAF1 complex and then exhibit in synergy with cisplatin to inhibit cell proliferation in cisplatin-resistant non-small cell lung cancer (NSCLC) cells. In addition, pimozide induces degradation of USP1 substrate ID1 and therefore attenuates leukemic cell growth. ML323 exerts excellent selectivity for DUBs. In non-small cell lung cancer and osteosarcomas, ML323 potentiates cisplatin-induced cytotoxicity. ML323-mediated inhibition of USP1 inhibits deubiquitylation of Fanconi anemia group D2 protein (FANCD2) and proliferating cell nuclear antigen (PCNA) and compromises translesion synthesis (TLS) and Fanconi anemia (FA) pathways. Thus, ML323 may provide a means to sensitize cancer cells to platinum-based therapies.

The small molecule inhibitor WP1130, also known as Degrasyn, is derived from a compound with JAK2 (the Janus kinase 2) kinase inhibitory activity. WP1130 rapidly induces accumulation of polyubiquitylated proteins, resulting in induction of apoptosis. WP1130 is a partially selective inhibitor that directly inhibits the deubiquitylating activity of USP9X, USP5, and USP14, all of which regulate survival protein stability and proteasome function. In particular, WP1130mediated USP9X inhibition induces apoptosis by reducing MCL-1 levels and increasing tumor cell sensitivity to chemotherapy. Moreover, USP9X inhibition by WP1130 can inhibit the growth of ERG-positive tumors in vitro and in mouse xenograft models of prostate cancer. 


\section{References}

Dou QP, Zonder JA (2014) Overview of proteasome inhibitor-based anti-cancer therapies: perspective on Bortezomib and second generation proteasome inhibitors versus future generation inhibitors of ubiquitin proteasome system. Curr Cancer Drug Targets 14:517-536

Farshi P, Rahul R, Deshmukh RR et al (2015) Deubiquitinases (DUBs) and DUB inhibitors: a patent review. Expert Opin Ther Pat 25:1191-1208

Fraile JM, Quesada V, Rodríguez D et al (2012) Deubiquitinases in cancer: new functions and therapeutic options. Oncogene 31:2373-2388

Harhaj EW, Dixit VM (2011) Deubiquitinases in the regulation of NF- $\mathrm{\kappa B}$ signaling. Cell Res 21:22-39

Harrigan JA, Jacq X, Martin NM et al (2018) Deubiquitylating enzymes and drug discovery: emerging opportunities. Nat Rev Drug Discov 17:57-78

He M, Zhou Z, Wu G et al (2017) Emerging role of DUBs in tumor metastasis and apoptosis: therapeutic implication. Pharmacol Ther 177:96-107

Iyengar PV (2017) Regulation of ubiquitin enzymes in the TGF- $\beta$ pathway. Int J Mol Sci 18:877

Komander D, Clague MJ, Urbé S (2009) Breaking the chains: structure and function of the deubiquitinases. Nat Rev Mol Cell Biol 10:550-563

Lim KH, Ramakrishna S, Baek KH (2013) Molecular mechanisms and functions of cytokine-inducible deubiquitinating enzymes. Cytokine Growth Factor Rev 24:427-431

Ndubaku C, Tsui V (2014) Inhibiting the deubiquitinating enzymes (DUBs). J Med Chem 58:1581-1595 